
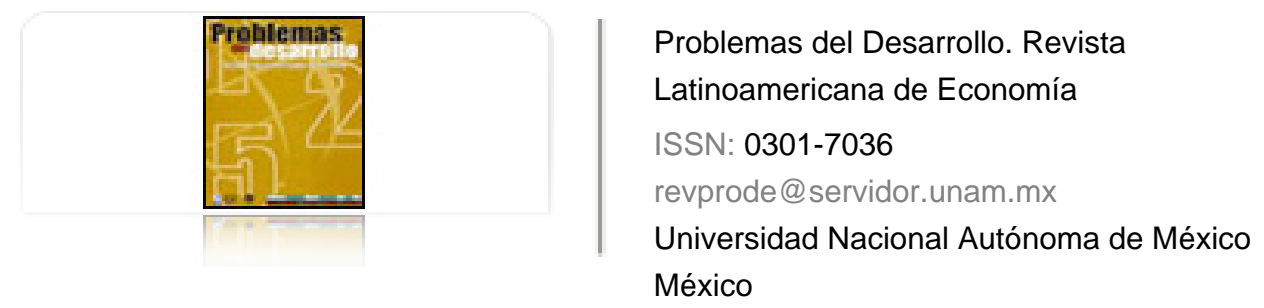

Luengo, Fernando

INDUSTRIA DE BIENES DE EQUIPO DE LA ECONOMÍA ESPAÑOLA: INSERCIÓN COMERCIAL Y CAMBIO ESTRUCTURAL

Problemas del Desarrollo. Revista Latinoamericana de Economía, vol. 42, núm. 167, octubrediciembre, 2011, pp. 123-145

Universidad Nacional Autónoma de México

Distrito Federal, México

Disponible en: http://www.redalyc.org/articulo.oa?id=11820101006

Cómo citar el artículo

- Número completo

- Más información del artículo

Página de la revista en redalyc.org

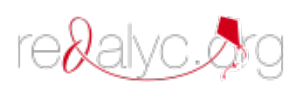

Sistema de Información Científica

Red de Revistas Científicas de América Latina, el Caribe, España y Portugal Proyecto académico sin fines de lucro, desarrollado bajo la iniciativa de acceso abierto 
Revista Problemas del Desarrollo, 167 (42), octubre-diciembre 2011

\title{
INDUSTRIA DE BIENES DE EQUIPO \\ DE LA ECONOMÍA ESPAÑOLA: INSERCIÓN COMERCIAL Y CAMBIO ESTRUCTURAL
}

\author{
Fernando Luengo*
}

Fecha de recepción: 20 de enero de 2011. Fecha de aceptación: 26 de mayo de 2011.

\section{RESUMEN}

El texto examina el comercio exterior de la industria de bienes de equipo de la economía española en el entorno comunitario. Dicho análisis se realiza a partir de la evolución de un conjunto de indicadores reveladores de la calidad de la inserción comercial: el nivel tecnológico y la posición de gama. La principal conclusión es que, aun aceptando la diversidad de posiciones y trayectorias observadas en la industria, parece que ésta ocupa un espacio relativamente subordinado, instalada en segmentos de mercado de mediabaja tecnología y gama.

Palabras clave: industria, bienes de equipo, competitividad, inserción comercial, cambio estructural, economía española.

\section{EQUIPMENT GOODS INDUSTRY IN THE SPANISH ECONOMY: COMMERCIAL INSERTION AND STRUCTURAL CHANGE}

\begin{abstract}
The study examines foreign trade for the equipment goods industry in the Spanish economy at a community level. The analysis is based on the movement of a combination of indicators that reveal the quality of trade insertion: the technological level and the position of the range. The principal conclusion is that, even accepting the diversity of positions and trajectories observed in the industry, it seems to occupy a relatively subordinate space, installed in market segments of medium-to-low technology and range. Key words: industry, equipment goods, competitiveness, trade insertion, structural change, Spanish economy.
\end{abstract}

* Profesor titular de la Universidad Complutense de Madrid. Correo electrónico: fluengoe@ ccee.ucm.es 
Fernando Luengo

\section{INDUSTRIE DE BIENS D'ÉQUIPEMENTS DE L'INDUSTRIE ESPAGNOLE : INSERTION COMMERCIALE ET CHANGEMENT STRUCTUREL \\ Résumé}

Ce texte fait l'examen du commerce extérieur de l'industrie de biens d'équipements de l'économie espagnole dans le contexte communautaire. Cette analyse est effectuée à partir de l'évolution d'un ensemble d'indicateurs révélateurs de la qualité de l'insertion commerciale : le niveau technologique et le niveau de gamme. La principale conclusion est que, même en acceptant la diversité de niveaux de gamme et trajectoires observées dans cette industrie, il semble qu'elle occupe une place relativement subordonnée, installée dans des segments de marché de faible technologie et de bas de gamme.

Mots clés : industrie, biens d'équipements, compétitivité, insertion commerciale, changement structurel, économie espagnole

\section{INDÚSTRIA DE BENS DE EQUIPAMENTO DA ECONOMIA ESPANHOLA: INSERÇÃO COMERCIAL E MUDANÇA ESTRUTURAL}

Resumo

O texto examina o comercio exterior da indústria de bens de equipamento da economia espanhola no entorno comunitário. Dita analise se realiza a partir da evoluçáo de um conjunto de indicadores reveladores da qualidade da inserçáo comercial: o nível tecnológico e a posiçáo relativa. A principal conclusáo é que, ainda aceitando a diversidade de posiçóes e trajetórias observadas na indústria, parece que esta ocupa um espaço relativamente subordinado, instalada em segmentos de mercado de media-baixa tecnologia. Palavras-chave: indústria, bens de equipamento, competitividade, inserção comercial, mudança estrutural, economia espanhola.

\section{資本貨物的西班牙經濟行業：貿易一體化和結構變化 摘要 \\ 該文探討了在西班牙經濟中的資本品行業在社區環境對外貿 易。這一分析是基於一套指標演變揭示了貿易一體化的質量: 技術水平和範圍的位置。主要的結論是，即使接受的立場和在 業界觀察軌跡的多樣性，似乎它佔據一個相對從屬的，市場上 中低技術和範圍安裝的分部}

關鍵詞：業，資本貨物，競爭力，貿易一體化，結構變化，西 班牙經濟 


\section{INTRODUCCIÓN}

Las líneas que siguen pretenden analizar el proceso de cambio estructural de la industria de bienes de equipo (IBE) en el contexto de la Unión Europa (UE). Conscientes de que cabe acometer dicho análisis desde muy diferentes perspectivas (posiblemente, todas ellas necesarias para capturar las transformaciones de calado en toda su complejidad y alcance), se trata de identificar las debilidades y fortalezas competitivas de esta industria, así como el carácter defensivo u ofensivo de la reestructuración operada, a partir de la evolución de un conjunto de ratios referidos al comercio exterior.

Situado en este ámbito, el objetivo del estudio es poner de manifiesto el contenido y la calidad de su inserción comercial en el mercado comunitario, trabajo que se aborda desde una doble perspectiva: los niveles tecnológicos y la escala de gama. La intersección de ambos planos dará la medida del posicionamiento estructural de la IBE.

\section{PLANTEAMIENTO GENERAL}

El espacio europeo se caracteriza por su diversidad, por acoger en su seno a economías estructuralmente diferentes (Commission of the European Communities, 2009; Gambarotto y Solari, 2008; Directorate General for Enterprise and Industrie, 2009; Aiginger y Davis, 2004; Davis, 2004). Esta diversidad -agravada, pero no causada, por las sucesivas incorporaciones a la UE de un nutrido grupo de países procedentes del mundo postcomunista- que ha permanecido e incluso se ha acentuado en un contexto en el cual se cerraba parcialmente la brecha nominal (proceso que la crisis económica ha detenido, primero, y ha invertido, después), objetivo central del Tratado de Maastricht y de las políticas económicas llevadas a cabo desde entonces (reducción de la inflación, el déficit, los tipos de interés y la deuda pública).

En la dinámica de la construcción europea han convivido, se han entrelazado en el mismo espacio económico, procesos convergentes y divergentes. Esa convivencia, rasgo estructural de la dinámica comunitaria, ahora merece una mayor atención para dar cuenta de las diferentes potencialidades de crecimiento económico, de la desigual capacidad de respuesta de las economías europeas ante el surgimiento de choques asimétricos y como posible explicación, entre otros factores, de la actual crisis económica (Lapavitsas et al, 2010). En este sentido, las asimetrías - productivas, tecnológicas, comerciales, distribución del ingreso- que han impregnado la dinámica comunitaria proporcionan, junto a la 
naturaleza sistémica del desorden financiero, un marco analítico de trascendental importancia para comprender la dimensión estructural de la crisis, la cual trasciende con claridad los avatares y el territorio de la mera coyuntura.

Abordar el análisis de una Europa estructuralmente diversa y jerarquizada, con una variedad de capitalismos que presentan rasgos bien distintos entre sí, precisa de propuestas teóricas y proyectos empíricos muy distintos de los que han inspirado el paradigma de la convergencia (Gambarotto y Solari, 2008; Beeson, Islam, 2004). Éste, basado en que el denominado proyecto europeo, con su combinación de lógicas mercantiles y acciones institucionales, homogeneizará las condiciones económicas de los países integrantes de la UE, cerrando de manera progresiva las brechas en el PIB por habitante, no parece capaz de dar cuenta de la complejidad de dinámicas y de las asimetrías que atraviesan el espacio comunitario.

El estudio de esta diversidad estructural admite (requiere, incluso) mirar en muy diferentes planos: los países y las regiones, los grupos sociales, los entornos institucionales, las configuraciones tecnológicas o las especializaciones productivas. También las modalidades de inserción comercial (el objeto de este texto), estrechamente relacionadas con la calidad de los tejidos empresariales y productivos (Mickiewicz, 2001; Radosevic y Rozeik, 2005; Fontagné, 2004; Landesmann, 2008).

El enfoque desarrollado en este trabajo se inscribe en una propuesta teórica que sitúa en el centro del análisis la segmentación de los mercados, en los que prevalece la competencia imperfecta, la información asimétrica y los rendimientos crecientes de escala (muy lejos, por lo tanto, de los enunciados neoclásicos que contemplan mercados homogéneos, libres de barreras a la entrada y salida, y con rendimientos constantes de escala). En este contexto, emergen factores de competitividad más complejos y sofisticados que los que se derivan del precio y el coste (Szalavetz, 2003).

El estudio que se presenta a continuación, basado en los niveles tecnológicos y las posiciones de gama pretende dar cuenta, justamente, de ese proceso de segmentación y de las dinámicas estructurales que alimenta. Las ventajas de competir en mercados que discriminan en función de la calidad -caracterizados por elevados grados de diferenciación y por absorber bienes difícilmente imitables- es que agregan a los precios-mecanismo de competencia dominante en los segmentos más estandarizados- otros elementos que dotan de singularidad a los bienes y servicios. Los productos instalados en dichos mercados no sólo presentan menor vulnerabilidad a la competencia procedente de las economías de bajos salarios, sino que, más relevante desde el punto de vista del cambio estructural, se benefician del dinamismo que deriva del progreso técnico y 
de la obtención de mejoras en la calidad de la oferta (Economic Commission for Europe, 2002). El texto conecta así con aquellos planteamientos orientados sobre todo a mostrar los aspectos estructurales de la integración comercial y que enfatizan, antes que la cantidad, la calidad de la inserción externa.

Buena parte de los estudios al respecto de la competitividad estructural en la esfera del comercio internacional han tomado como unidad de análisis el sector, la industria y los capítulos arancelarios. Esta ha sido la base para la elaboración de diferentes indicadores reveladores de las fortalezas y debilidades de las economías y de su posición competitiva, que proporciona una información útil, que resulta, sin embargo, insuficiente para el propósito de este trabajo, por excesivamente global e imprecisa.

La superación de esa restricción ha promovido diferentes investigaciones donde el núcleo analítico es el producto y la tarea (Grossman y Rossi-Hansberg, 2006; Landesmann y Wörz, 2006; United Nations Industrial Development Organization, 2009). En ellas subyace la idea de que las categorías anteriores (sector, industria, capítulo arancelario) presentan un nivel de agregación que dificulta la captura de la diversidad (tecnológica, de gama) de las actividades que agrupan. En línea con esas consideraciones, el análisis que se lleva a cabo en esta sección toma como base de partida las posiciones arancelarias con un nivel de desagregación de 6 dígitos; mínimo nivel requerido para que las comparaciones internacionales (en nuestro caso, España con respecto a la UE I 5), en el ámbito de la inserción comercial, tengan virtualidad interpretativa.

La tecnología ha sido uno de los aspectos que ha articulado los trabajos al respecto de la competitividad estructural y la segmentación de los mercados (Economic Commission for Europe, 2002; Lall, 2002; Fontagné, 1999; Cooper, 1995). Las empresas y las naciones que ocupan posiciones de supremacía en la esfera tecnológica -concepto que, en un sentido amplio, comprende tanto el conocimiento incorporado en las maquinas y bienes de equipo como cada vez más, los intangibles- se instalan en mercados gobernados por criterios competitivos distintos del precio, como la calidad, la variedad y la renovación del surtido productivo o la prestación de servicios al cliente.

Cabe realizar, no obstante, dos precisiones al respecto. La primera, tiene que ver con que algunas de las actividades de medio o aun bajo perfil tecnológico acreditan un indudable potencial de crecimiento, pudiendo generar un alto valor añadido por la vía de aminorar los costes de fabricación. La segunda, alude a la heterogeneidad de las denominadas ramas tecnológicas, donde conviven producciones instaladas en mercados de desigual complejidad y sofisticación. Todo ello invita a la prudencia a la hora de asociar de manera automática nivel tecnológico con competitividad. 
El trabajo empírico desarrollado a partir de clasificaciones tecnológicas sustentadas en la industria, o en los grandes agregados arancelarios, no parece adecuado para enfrentar las restricciones que acaban de señalarse. Por esa razón, se ha adoptado la taxonomía propuesta por la Comisión Económica para América Latina y El Caribe (CEPAL) donde se identifican los flujos comerciales conforme a su nivel tecnológico (alto, medio y bajo), también con un nivel de desagregación de seis dígitos de la nomenclatura arancelaria.

En paralelo a la elaboración de una clasificación tecnológica a partir de los productos exportados e importados, otras investigaciones se han ocupado de definir la posición de gama de los flujos comerciales, utilizando el nivel y la evolución de los valores unitarios (vu), (esto es, el cociente entre el valor de las exportaciones o importaciones y la cantidad, medida en kilogramos o toneladas) a partir de la comparación de esta ratio en diferentes países (Graziani, 2002).

La hipótesis que sustenta estas investigaciones es que los precios (los vu nos aproximan a ellos, pues esa información no está disponible en las estadísticas de aduanas) son un indicio, un indicador aproximado de la calidad. Al comparar productos similares con un nivel de desagregación suficiente (España y la UEI 5), un vU superior pondría de manifiesto que el comprador ha abonado un precio más elevado, justificado por el diferencial de calidad de ese producto (Aiginger, 2000).

Los autores que han trabajado en esta dirección sugieren, en todo caso, una interpretación prudente de los resultados obtenidos. Por un lado, el aumento en los vu, para que refleje un mejor posicionamiento en segmentos de gama más sofisticados, no debe traducirse en una pérdida de cuota de mercado; en este caso, dicho aumento podría deberse a un encarecimiento en los costes de producción, trasladado a los precios finales, que habría recibido la correspondiente penalización de la demanda. Se señala, de otra parte, que el vu (indicador que, no se olvide, se sitúa en el territorio de los precios) no puede capturar, por definición, otros factores como por ejemplo, la calidad de las redes comerciales o la efectividad de las estrategias publicitarias, aspectos tan relevantes como las características intrínsecas de los productos que son depositados en los mercados.

Una precisión añadida sobre el vínculo entre tecnología y gama en la línea de los trabajos desarrollados por el Centre d'Études Prospectives et d'Informations Internationales (CEPII) (Fontagné, 1999; Mulder, Rodrigo y Zignago, 2009; Gaulier y Zignago, 2008). En cada uno de los niveles tecnológicos, cabe encontrar distintas gamas de productos, y a la inversa, en cada una de las escalas de gama es posible situar distintos segmentos tecnológicos. De este modo, teniendo en cuenta las variadas combinaciones tecnología-gama se puede dibujar un complejo mapa de competitividad estructural, donde las mejores posiciones 
competitivas resultan de la intersección de los subconjuntos "alta tecnología-alta gama", mientras que las mayores debilidades se localizan en el cruce de los subconjuntos "baja gama-baja tecnología". Además de localizar estos lugares extremos, mostrar la franja de "colores intermedios" en la que caben diferentes combinaciones de tecnología y gama.

\section{ASPECTOS METODOLÓGICOS}

Para captar el posicionamiento estructural de la IBE en los años de crisis y su evolución, se comparan dos subperiodos: el bienio 2008/2009 y los ańos inmediatamente anteriores (2004/2007). Se consideran los datos promedio de ambos tramos. El espacio de referencia para el que se realiza el estudio es la UE 27 (se comparan las posiciones de España y de la UE I 5), mercado fundamental para el conjunto de la industria española, donde se realiza la mayor parte de nuestro comercio exterior. Téngase en cuenta que al adoptar la UE I 5 como base de comparación de la posición comercial de la IBE española tomamos como referencia una zona caracterizada por una induable heterogeneidad a la que pertenecen países con muy distinta renta por habitante. En trabajos posteriores será necesario abordar análisis más específicos donde se sitúe a la IBE de España frente a cada una de las economías que rivalizan con nosotros en el espacio comunitario.

La información estadística sobre las transacciones comerciales procede de Eurostat (COMEXT). Se ha utilizado la nomenclatura armonizada con un nivel de desagregación de seis dígitos, lo que supone 916 ítem arancelarios (de los 1056 considerados por el Ministerio de Industria, Comercio y Turismo), que representan entre $95 \%$ y $99 \%$ del total de exportaciones de BE, dependiendo del periodo. La diferencia consiste en los productos que han sido suprimidos, bien por falta de datos o porque presentan valores anómalos que distorsionaban el conjunto de la serie (Cuadro 1).

Para la obtención de datos agregados, se ha procedido a ponderar cada uno de esos item por la importancia relativa de las exportaciones con respecto a valor global. El análisis de la posición de gama se realiza a partir del vu de esta manera:

Se obtiene así un mapa de valores que se agrupan siguiendo tres criterios. Los resultados inferiores a 0.85 representan a los productos de baja gama; por encima de 1.15 se encuentran los bienes de gama más elevada; mientras que los productos de gama media se encuentran en el intervalo 0.85-1.15.

Para calibrar las tendencias estructurales (permanencia o variabilidad de las mismas) a lo largo del tiempo, se compara para cada producto los vu español y comunitario en 2004/2007 y 2008/2009. Se supone que hay un cambio 
Fernando Luengo

Cuadro 1. Número de productos según tipo de tecnología y posición de gama

\begin{tabular}{lrrrrrr} 
& \multicolumn{6}{c}{ Gama } \\
\cline { 2 - 7 } & Alta & Media & Baja & Alta & Media & Baja \\
\cline { 2 - 7 } \multicolumn{1}{c}{ Tecnología } & 63 & 46 & 87 & 38 & 28 & 130 \\
\hline Alta & 151 & 150 & 233 & 109 & 82 & 343 \\
Media & 60 & 61 & 49 & 47 & 48 & 75 \\
Baja & 7 & 3 & 6 & 7 & 3 & 6 \\
Recursos Naturales & 7007 &
\end{tabular}

Fuente: Elaboración propia a partir de COMEXT.

Cuadro 2. Explicación de la elaboración del índice de gama.

$V U i, p=\frac{X i, p}{Q i, p}$
X: exportaciones
Q: cantidades exportadas, expresadas en Kilos
I: producto
P: país
La posición de gama se calcula a partir de:
PGi, $=\frac{V U i, p}{V U i, u e}$
Donde: UE es el conjunto de la Unión Europea

estructural si se altera, en una proporción sustancial, la proporción entre los vU, mientras que si dicha proporción se mantiene aproximadaente estable se estaría ante un escenario de relativa inercia estructural (si, en un supuesto extremo, la ratio no experimentase cambio alguno la nube de puntos coincidiría exactamente con la bisectriz). De manera estilizada, cabe contemplar cuatro escenarios: inercial, progresión, rezago y alteración (Gráfica 1).

Al comparar los vu de las dos zonas (España y la UE I 5 ) los datos se agrupan alrededor de cuatro cuadrantes (Gráfica 2). Los dos superiores ponen de manifiesto fortalezas estructurales, mientras que los inferiores sugieren carencias. La posición con respecto a la bisectriz refleja la intensidad del cambio operado, tanto en los registros positivos como en los negativos.

Si se compara la variación de los vu y las cuotas exportadoras se obtiene igualmente una Gráfica que ilustra cuatro recorridos distintos. Las ganancias de 
Gráfica 1 Tendencias estructurales en función del comportamiento de los valores unitarios

$$
\text { Brusca alteración estructural }
$$

$$
\text { Inercia estructural }
$$
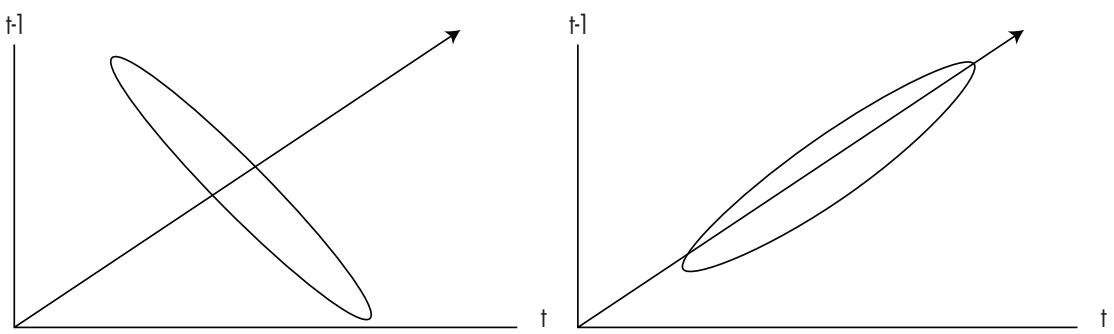

$\oplus$
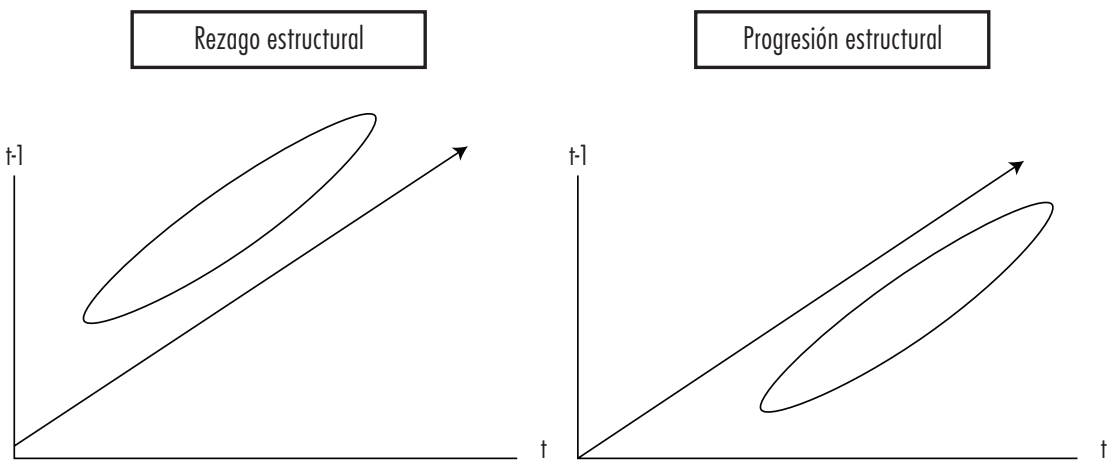

Gráfica 2. Comparación de los valores unitarios (en logaritmos)

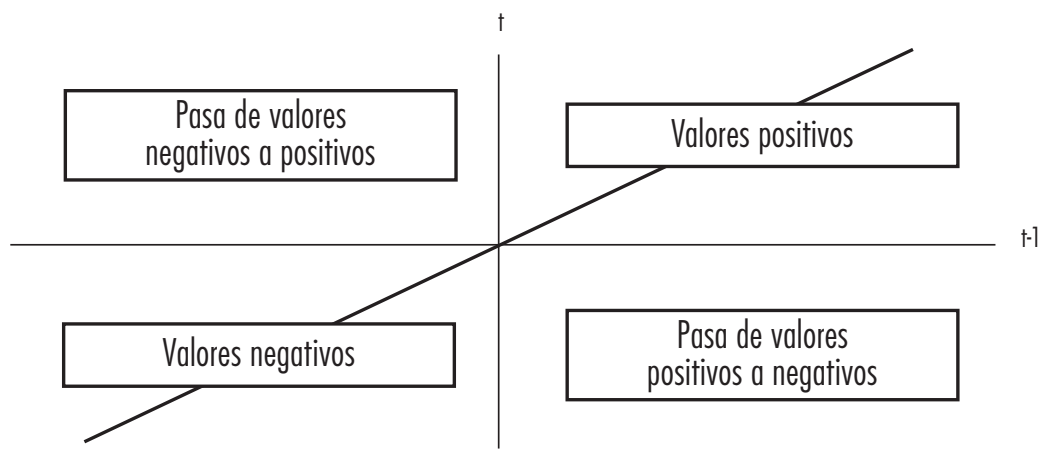

131

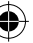


Fernando Luengo

cuota se sitúan en los cuadrantes primero, donde aumentan asimismo los vU, y tercero, donde éstos retroceden. Las pérdidas de cuota, acompañadas de incrementos en los vu se muestran en el cuadrante segundo, en tanto que el escenario más desfavorable, se dibuja en el cuadrante cuarto (Gráfica 3).

\section{DINÁMICA ESTRUCTURAL E INSERCIÓN COMERCIAL}

Las Gráficas 4 y 5 ofrecen una perspectiva general de la trayectoria seguida por los vu en las diferentes categorías tecnológicas. Para ello se ha ponderado cada uno de esos valores (en Espańa y en el conjunto de la UE) por el peso relativo del correspondiente ítem en las exportaciones de cada nivel tecnológico y en el conjunto de las de bienes de equipo.

Dos conclusiones surgen al respecto en esta primera aproximación sobre la calidad de la inserción comercial de la IBE. La primera es que nuestra industria se encuentra situada en segmentos de gama inferiores a los comunitarios. Esta situación es aplicable a los tres tramos tecnológicos, alto, medio y bajo. La brecha es más pronunciada justamente en los productos de mayor sofisticación. La segunda es que la eclosión de la crisis ha ensanchado aún más esa distancia, también en todas las categorías tecnológicas. Si bien más adelante se realizan algunas matizaciones al respecto, parece que los últimos años han representado una intensa presión sobre los precios de nuestras exportaciones en el mercado comunitario, superior a la registrada por los países de nuestro entorno.

En la estructura exportadora de nuestra industria dominan con claridad los productos de media tecnología (Cuadro 2) -más del 60\% de las ventas totales-,

Gráica 3. Valores unitarios (en logaritmos) y cuotas exportadoras (en porcentaje)

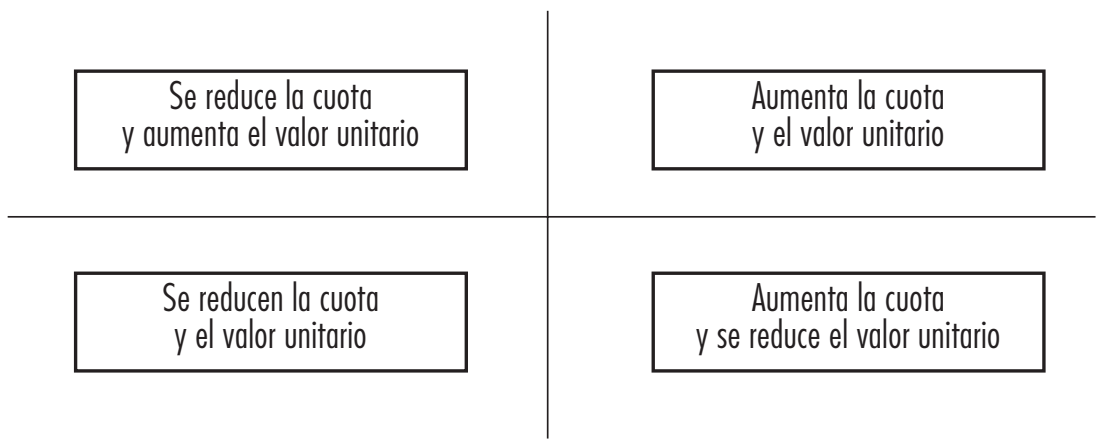


Gráfica 4. Posición de gama en la Industria de Bienes de Equipo según nivel tecnológico ${ }^{*}$ )

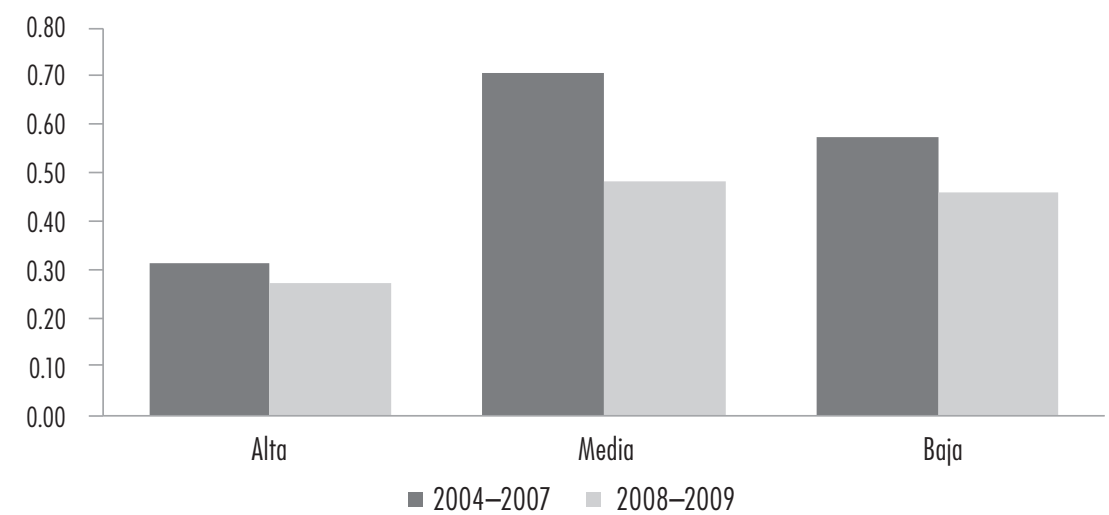

* Factor de ponderación: peso relativo de las exportaciones de cada producto con respecto al total de cada nivel tecnológico. Fuente: Elaboración propia con datos de Comext.

Gráfica 5. Posición de gama en la Industria de Bienes de Equipo según nivel tecnológico (*)

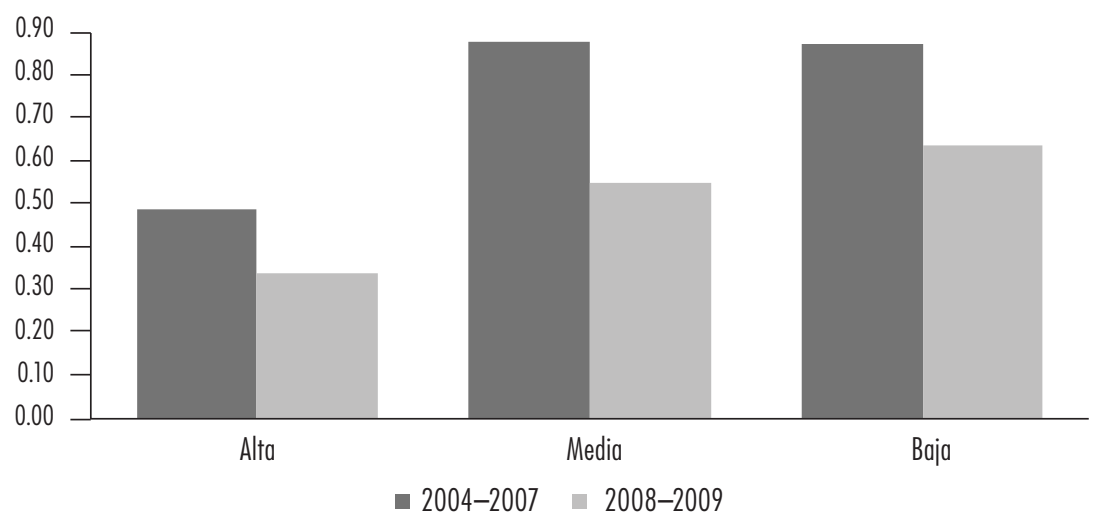

* Factor de ponderación: peso relativo de las exportaciones de cada producto con respecto al total de las ventas de bienes de equipo.

Fuente: Ob. cit.

porcentaje que retrocede ligeramente entre ambos subperiodos, retroceso que se compensa por las subidas registradas en las categorías de alta y baja tecnología. En cuanto a la importancia relativa de los tres rubros en las exportaciones comunitarias (Cuadro 3), los porcentajes se distribuyen de manera más equilibrada (entre 11 y 14\%) registrándose progresos en los de media y alta tecnología. 
Fernando Luengo

Ambos cuadros ofrecen información sobre las combinaciones tecnologíagama. Acaso el hecho más sobresaliente sea que el aumento en el peso relativo de nuestras exportaciones, en los tres segmentos tecnológicos, se haya producido en las escalas de gama inferiores. Los productos de gama media, en el tramo tecnológico inferior, son los que han experimentado una clara evolución al alza, registrándose un vuelco considerable en las proporciones. ${ }^{1}$ Desde la perspectiva de las cuotas de mercado, las tendencias son algo distintas. En este caso, los productos de alta y media gama, en los tramos de tecnología baja y más sofisticada, han aumentado su relevancia.

Cuadro 3. Tecnología y gama (porcentaje de las exportaciones de Bienes de Equipo)

\begin{tabular}{lrrrrrrrr}
\hline & \multicolumn{9}{c}{$2004-2007$} & \multicolumn{5}{c}{$2008-2009$} \\
\hline Tecnología/gama & \multicolumn{1}{c}{ Alta } & Media & \multicolumn{1}{c}{ Baja } & Total & \multicolumn{1}{c}{ Alta } & Media & Baja & Total \\
\hline Alta & $4.4 \%$ & $5.8 \%$ & $6.4 \%$ & $16.6 \%$ & $2.8 \%$ & $5.5 \%$ & $11.1 \%$ & $19.4 \%$ \\
Media & $14.1 \%$ & $36.3 \%$ & $15.8 \%$ & $66.1 \%$ & $13.0 \%$ & $10.2 \%$ & $38.7 \%$ & $62.0 \%$ \\
Baja & $2.0 \%$ & $10.0 \%$ & $4.6 \%$ & $16.7 \%$ & $1.9 \%$ & $8.0 \%$ & $8.2 \%$ & $18.1 \%$ \\
\hline
\end{tabular}

Fuente: Ibidem.

Cuadro 4. Tecnología y gama (porcentaje en las exportaciones de la UE)

\begin{tabular}{lcccccccc}
\hline & \multicolumn{3}{c}{$2004-2007$} & \multicolumn{5}{c}{$2008-2009$} \\
\hline Tecnología/gama & Alta & Media & Baja & Total & Alta & Media & Baja & Total \\
\hline Alta & $3.0 \%$ & $4.5 \%$ & $3.2 \%$ & $10.8 \%$ & $3.7 \%$ & $3.9 \%$ & $3.6 \%$ & $11.2 \%$ \\
Media & $2.7 \%$ & $5.8 \%$ & $3.0 \%$ & $11.6 \%$ & $5.1 \%$ & $3.7 \%$ & $3.7 \%$ & $12.6 \%$ \\
Baja & $3.5 \%$ & $4.9 \%$ & $5.7 \%$ & $14.1 \%$ & $2.9 \%$ & $5.8 \%$ & $4.7 \%$ & $13.4 \%$ \\
\hline
\end{tabular}

Fuente: Ibidem.

La distribución de las nubes de puntos y las correspondientes líneas de regresión en la Gráfica 6 confirman, como se señaló antes, que se ha operado un cierto ajuste de los precios relativos. Descartándose los dos escenarios extremos -inercia y brusca alteración-, parece prevalecer el que ha sido denominado

1 Variación imputable sobre todo a que la partida 870421 (vehículos de motor para el transporte de bienes) que representa entre el $13 \%$ y el $15 \%$ de las exportaciones, ha pasado de gama media a baja. 
rezago, siempre teniendo en cuenta que la casuística es ciertamente muy diversa. En efecto, como se puede apreciar en la Gráfica, una proporción sustancial de los puntos se sitúa a la izquierda de la bisectriz, como consecuencia de que los vu relativos de esos productos (el que resulta de comparar los respectivos ratios de nuestra economía con los de la uE entre 2004/2007 y 2008/2009) eran inferiores en el bienio de crisis que en el periodo anterior.

Esa evolución podría significar que, en un contexto de contracción de los mercados y de intensificación de la competencia, se ha asistido a un cierto ajuste en los precios a la baja, corrección que sería visible en los diferentes tramos tecnológicos, concentrándose sobre todo en aquellos bienes que acreditaban valores superiores.

Gráifa 6. Correlación de los valores unitarios (VUesp/Vuve; 2004/2007-2008/2009)
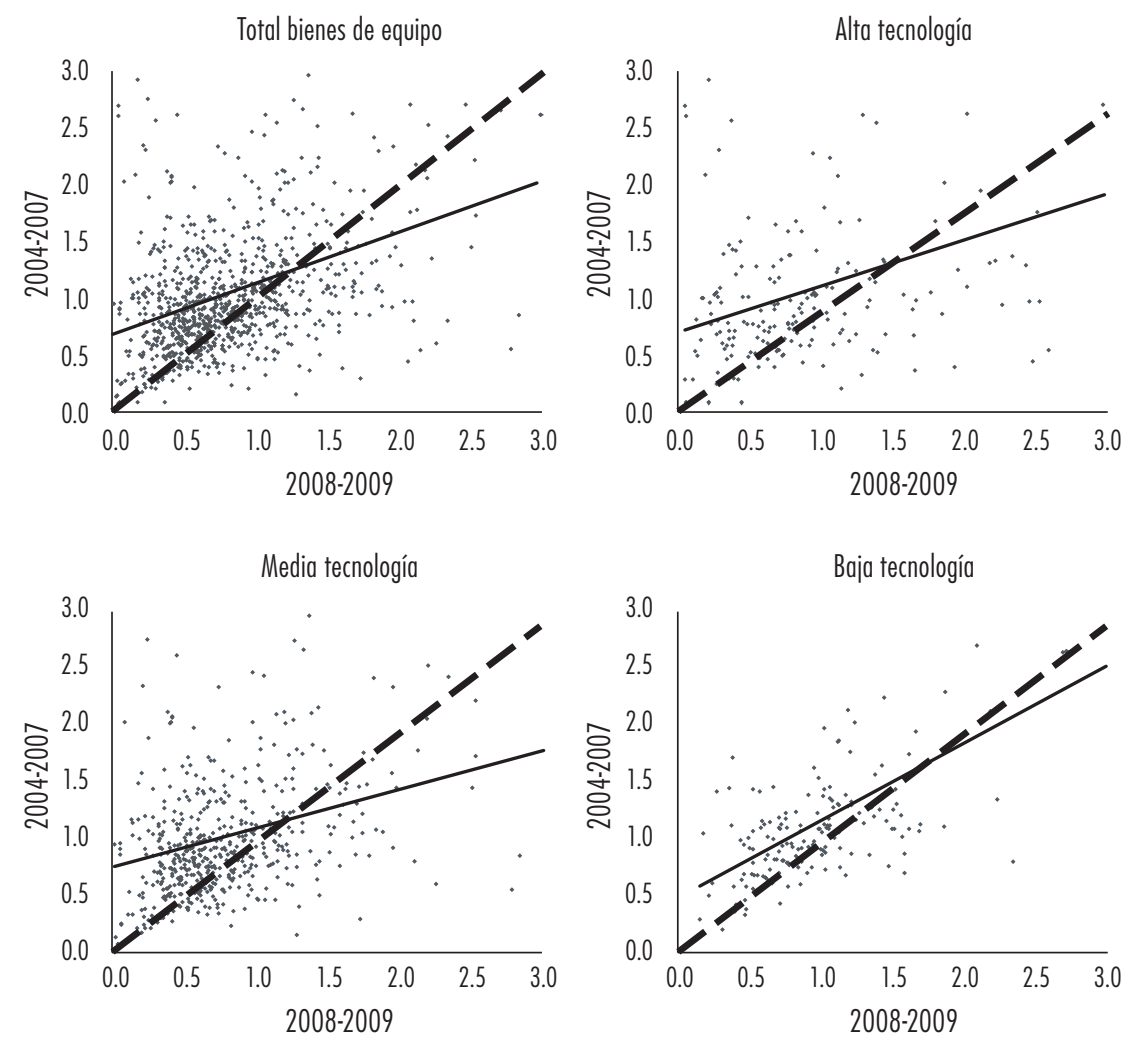

Fuente: Ibidem 
Fernando Luengo

Las siguientes gráficas relacionan la evolución de los vu de las exportaciones españolas con los de la UE en los subperiodos seleccionados. Al aplicarse el logaritmo, toman valores negativos aquellos productos que se exportan a un valor unitario menor que el promedio comunitario y positivos los que se encuentren en la situación opuesta. La información se presenta para el conjunto de la industria y para cada uno de los segmentos tecnológicos.

La línea discontinua representa la bisectriz. Los productos que se sitúen sobre ella no habrán visto modificada su posición de un periodo a otro. La línea negra es la recta de regresión de la muestra y, a partir de su comparación con la bisectriz, se puede razonar si se ha registrado un aumento, reducción o mantenimiento de los vu de exportación en relación con la UE. Además, se incluye información sobre el porcentaje de las exportaciones asociado a cada uno de los cuadrantes de las respectivas gráficas.

Gráfica 7. Valores unitarios de la Industria de Bienes de Equipo (Logaritmo de VUesp/VUue; 2004/2007-

2008/2009)

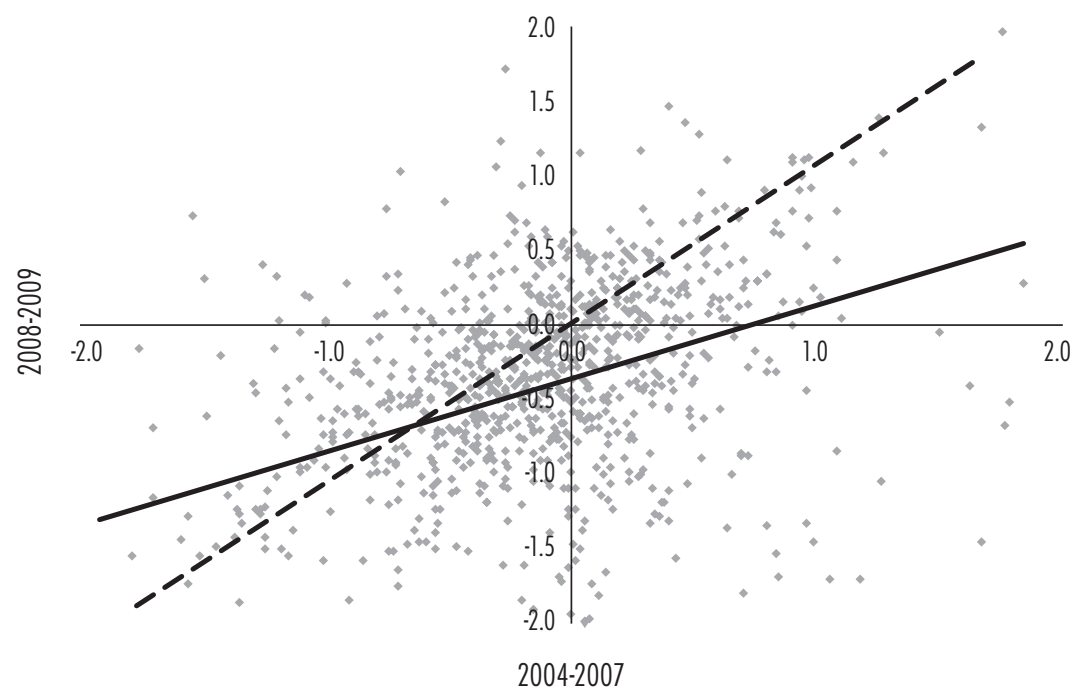

\begin{tabular}{cccccc}
\hline$N^{0}$ bienes & cuota 04-07 & cuota 08-09 & $N^{0}$ bienes & cuota 04-07 & cuota 08-09 \\
\hline 97 & $6.6 \%$ & $8.0 \%$ & 166 & $18.7 \%$ & $20.3 \%$ \\
459 & $59.0 \%$ & $56.6 \%$ & 194 & $15.7 \%$ & $15.1 \%$ \\
\hline
\end{tabular}

Fuente: Ibidem.

Nota: El cuadro inferior indica el número de bienes posicionado en cada cuadrante, así como sus respectivas cuotas de mercados. 
Gráfica 8. Valores unitarios de la IBE alto contenido tecnológico (Logaritmo de VUesp/Vuve; 2004/20072008/2009)

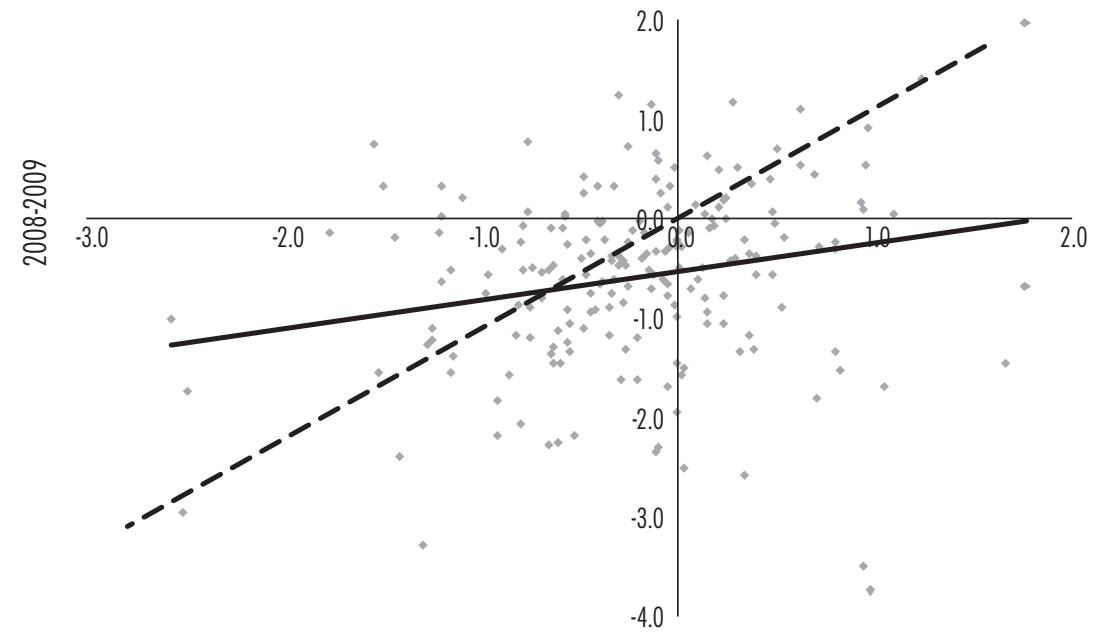

\begin{tabular}{ccccccc}
\multicolumn{7}{c}{$2004-2007$} \\
\hline & $N^{0}$ bienes & cuota 04-07 & cuota 08-09 & $N^{0}$ bienes & cuota 04-07 & cuota 08-09 \\
\hline & 23 & $0.8 \%$ & $1.4 \%$ & 23 & $2.0 \%$ & $3.4 \%$ \\
& 103 & $9.9 \%$ & $10.1 \%$ & 47 & $4.0 \%$ & $4.6 \%$ \\
Total & 196 & $16.64 \%$ & $19.43 \%$ & & & \\
\hline
\end{tabular}

Fuente: Ibidem

Nota: El cuadro inferior indica el número de bienes posicionado en cada cuadrante, así como sus respectivas cuotas de mercados.

En las gráficas se aprecia la distribución de la nube de puntos alrededor de los cuatro cuadrantes. Una primera observación general, que pone de manifiesto cierta fragilidad de nuestro perfil exportador, es que los productos situados en sendos cuadrantes superiores (con registros positivos en los vu en ambos subperiodos o que han transitado desde valores positivos a negativos) representaban el 28\% de las exportaciones en 2008/2009 (25\% en los ańos anteriores). Por el contrario, los productos que acreditan valores negativos o han pasado de registros positivos a negativos, absorbían en 2008/2009 el 74\% de las exportaciones, porcentaje sólo un punto porcentual inferior al obtenido en 2004/2007.

La mayor concentración, por encima del $50 \%$ de las exportaciones, se registra en el cuadrante inferior izquierdo, donde se anotan los pares de valores 
Fernando Luengo

Gráfica 9. Valores unitarios de la Industria de Bienes de Equipo de contenido tecnológico medio. (Logaritmo de VUesp/VUue; 2004/2007-2008/2009)

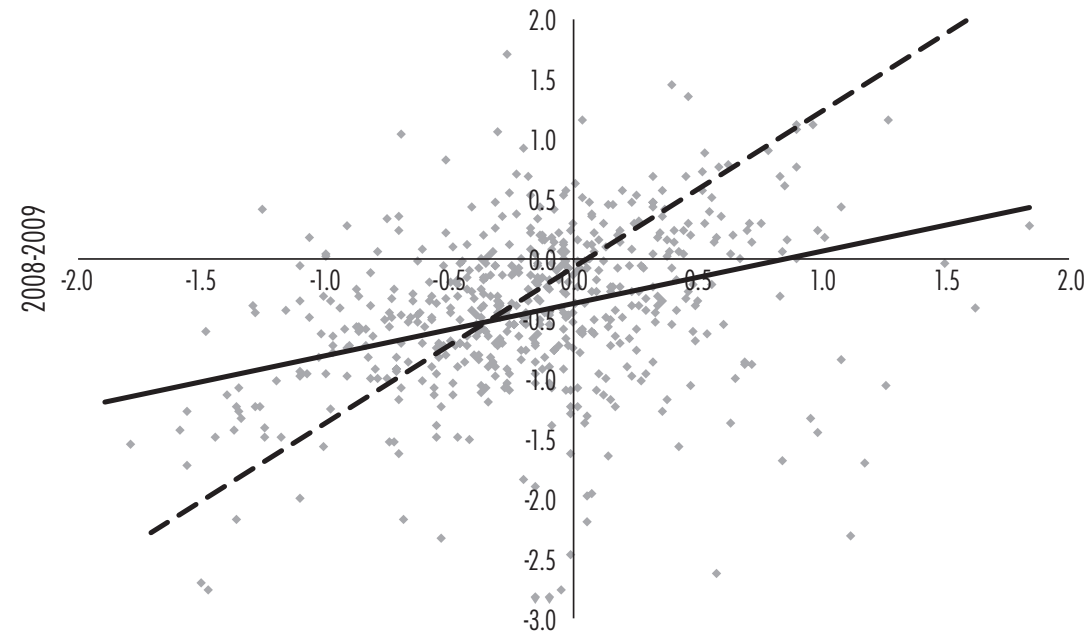

2004-2007

\begin{tabular}{ccccccc}
\hline & $N^{0}$ bienes & cuota 04-07 & cuota 08-09 & $N^{0}$ bienes & cuota 04-07 & cuota 08-09 \\
\hline & 54 & $3.6 \%$ & $4.3 \%$ & 81 & $11.3 \%$ & $10.8 \%$ \\
& 287 & $41.8 \%$ & $38.7 \%$ & 112 & $9.5 \%$ & $8.3 \%$ \\
Total & 534 & $66.18 \%$ & $61.99 \%$ & & & \\
\hline
\end{tabular}

Fuente: Ibidem.

Nota: El cuadro inferior indica el número de bienes posicionado en cada cuadrante, así como sus respectivas cuotas de mercados.

negativos; esto es, en los dos periodos examinados los valores de nuestros bienes se sitúan por debajo de los comunitarios. Se observa, no obstante, una ligera reducción en el referido porcentaje.

El cuadrante situado en la parte superior derecha contiene aquellos productos que, por el contrario, han presentado valores superiores a los comunitarios tanto en 2004/2007 como en 2008/2009. En este último periodo representaban una quinta parte aproximadamente de las ventas totales, con una suave tendencia ascendente.

Los productos que han visto cambiar su signo se encuentran en los otros dos cuadrantes. Los que han transitado desde posiciones negativas en 2004/2007 a positivas en el bienio de crisis representaban poco menos del $10 \%$ de las ventas 
totales en los últimos años, con tendencia a aumentar. En un sentido opuesto, los que han pasado de cifras positivas a negativas contribuían, con escasas variaciones, a generar el $15 \%$ de las exportaciones.

La pendiente ascendente de la línea de regresión y un relativamente alto coeficiente de correlación describe la existencia de cierta inercia estructural y que no se han registrado excesivas desviaciones intertemporales de los vu.

¿Se han mantenido estas pautas en las diferentes categorías tecnológicas? Los productos clasificados como de media tecnología son el componente fundamental de las ventas de esta industria (representan más del 60\% de las exportaciones, porcentaje que tiende a reducirse). Una diferencia relevante con respecto al conjunto del sector es que los bienes situados en el primer y cuarto cuadrantes

Gráica 10. Valores unitarios de la Industria de Bienes de Equipo de contenido tecnológico bajo (Logaritmo de VUesp/VUue; 2004/2007-2008/2009)

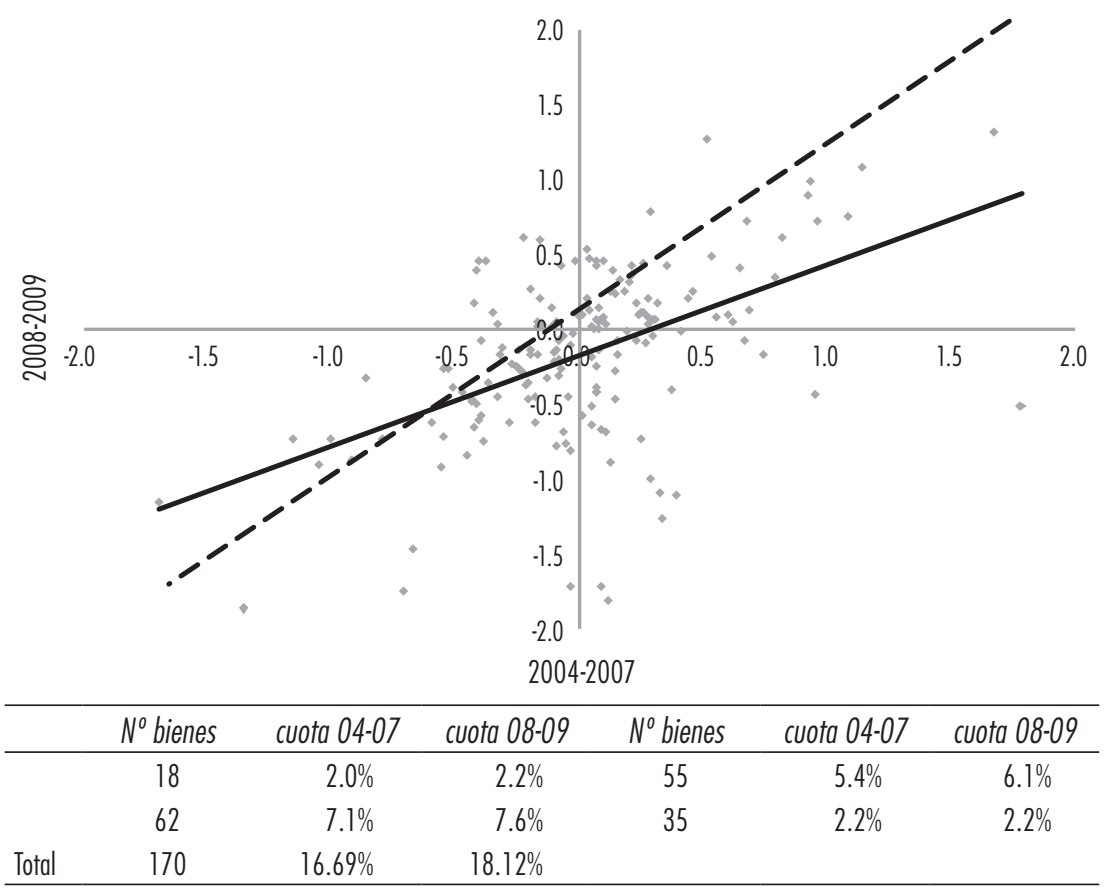

Fuente: Ibidem.

Nota: El cuadro inferior indica el número de bienes posicionado en cada cuadrante, así como sus respectivas cuotas de mercados. 
Fernando Luengo

han experimentado una reducción de su cuota exportadora. Los otros dos han seguido idéntico comportamiento que el conjunto de la industria. Tendencia básicamente distinta de la observada en los productos de mayor y menor calado tecnológico (los cuales han ganado peso relativo en las ventas exteriores de la industria). Con respecto a las cuotas exportadoras, se han registrado aumentos en los cuatro cuadrantes.

Las gráficas que se presentan a continuación, comparan las variaciones de los valores unitarios (expresados en logaritmos) y las cuotas de mercado, mostrando asimismo los correspondientes porcentajes de las ventas realizadas por la industria. Los productos donde se ha registrado una ganancia de cuota representaban en 2008/2009 el 46\% de las ventas totales, catorce puntos porcentuales más que en la etapa precedente. La situación más positiva es la reflejada en el cuadrante superior derecho, donde de manera simultánea aumentan los vu y las cuotas; el inferior derecho refleja un aumento de cuota en un contexto donde los vu se han reducido. Mientras que los primeros mostrarían, según la convención metodológica empleada en este trabajo, una mejora en la calidad, los segundos acreditarían una positiva elasticidad demanda-precio.

Gráfica 11. Valores unitarios y cuotas exportadoras en la Industria de Bienes de Equipo (Variación entre $2004 / 2007$ y 2008/2009)

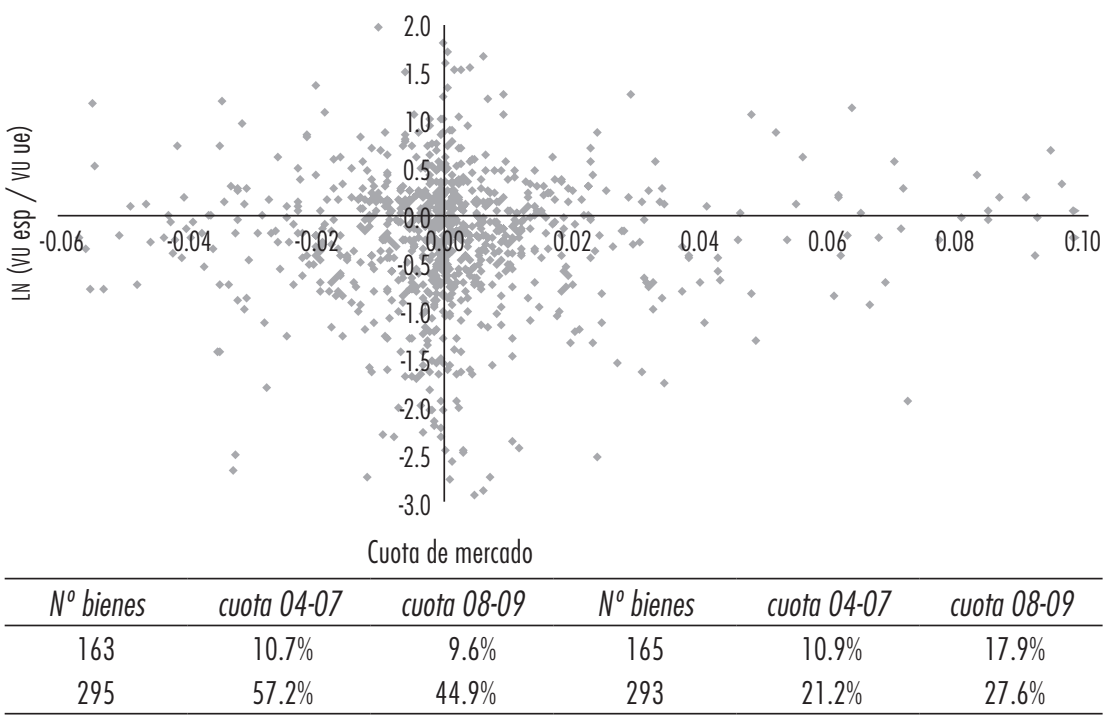

Fuente: Ibidem.

Nota: El cuadro inferior indica el número de bienes posicionado en cada cuadrante, así como sus respectivas cuotas de mercados. 
Gráica 12. Valor unitario y cuotas exportadoras en la Industria de Bienes de Equipo de alto contenido tecnológico (Variación entre 2004/2007 y 2008/2009)

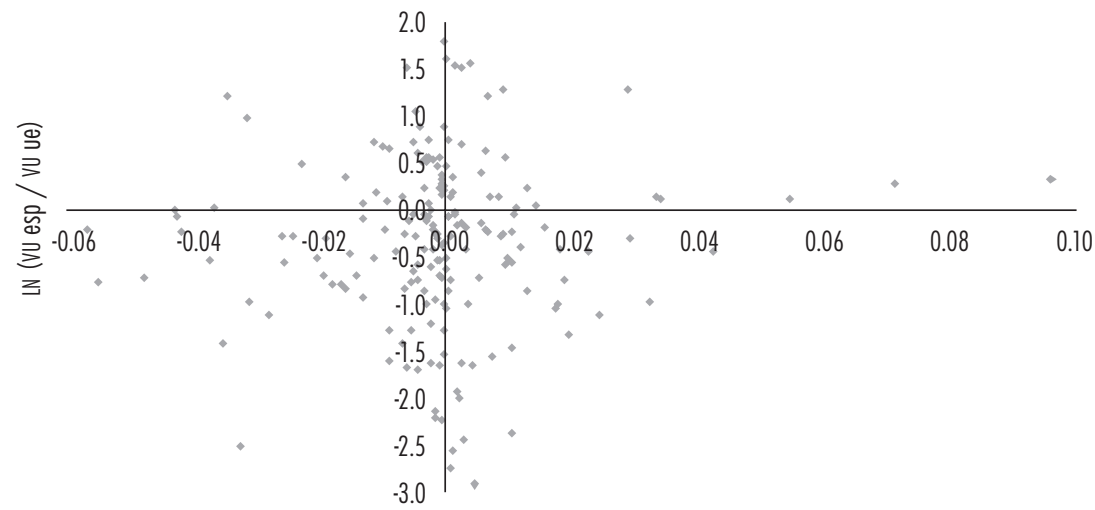

Cuota de mercado

\begin{tabular}{ccccccc}
\hline & $N^{0}$ bienes & cuota 04-07 & cuota 08-09 & $N^{0}$ bienes & cuota 04-07 & cuota 08-09 \\
\hline & 34 & $2.8 \%$ & $3.5 \%$ & 30 & $1.5 \%$ & $3.9 \%$ \\
& 75 & $7.7 \%$ & $6.3 \%$ & 57 & $4.6 \%$ & $5.7 \%$ \\
Total & 196 & $16.64 \%$ & $19.43 \%$ & & & \\
\hline
\end{tabular}

Fuente: Ibidem.

Nota: El cuadro inferior indica el número de bienes posicionado en cada cuadrante, así como sus respectivas cuotas de mercados.

La pérdida de cuota presenta el escenario más desfavorable. Los productos que se encuentran en esa situación, habiendo aumentado o disminuido los vu representaban en 2008/2009 el 55\% de las ventas de la industria, porcentaje que aún siendo importante, ha bajado de manera sustancial desde 2004/2007, cuando estaba situado en un $68 \%$.

El más relevante, desde el punto de vista cuantitativo, es aquel donde retroceden al mismo tiempo vu y cuotas (cuadrante inferior izquierdo), si bien, y este sería un aspecto positivo, se observa que estos productos pasaron a representar en los ańos de crisis una parte menor en las exportaciones totales de lo que suponían en el cuatrienio anterior. Finalmente, los bienes en los que ha aumentado el vu y, en paralelo, han visto cómo se reducía su cuota suponían el 10\% de las ventas totales, porcentaje algo menor al del periodo precedente.

Con desigual intensidad, se han registrado tendencias similares en los diferentes tramos tecnológicos, con la única excepción de los productos de mayor sofisticación. En este caso también aumenta la relevancia en la estructura 
Fernando Luengo

Gráfica 13. Valores unitarios y cuotas exportadoras en la Industria de Bienes de Equipo de contenido tecnológico medio (Variación entre 2004/2007 y 2008/2009)

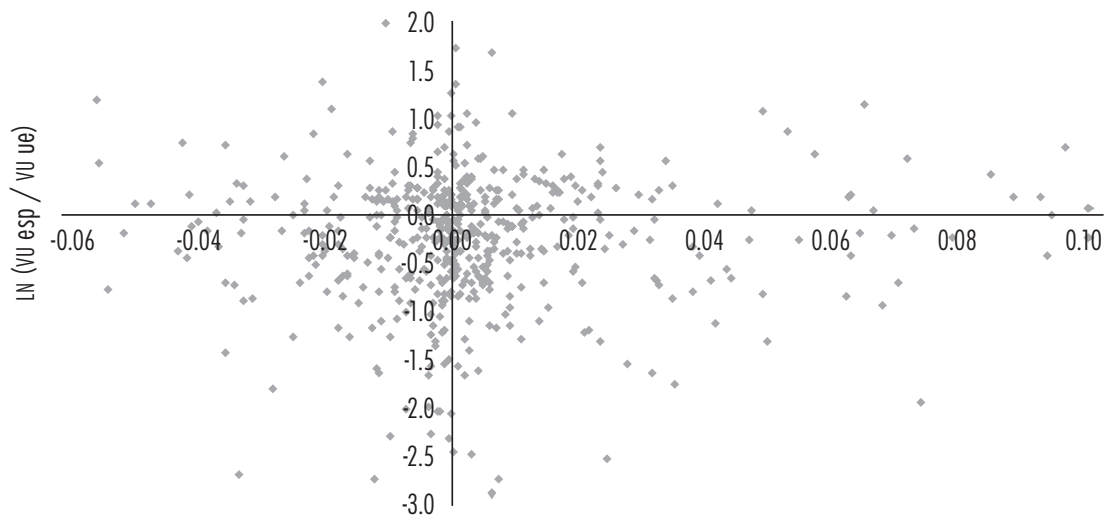

\begin{tabular}{ccccccc}
\multicolumn{8}{c}{ Cuota de mercado } \\
\hline & $N^{0}$ bienes & cuota 04-07 & cuota 08-09 & $N^{0}$ bienes & cuota 04-07 & cuota 08-09 \\
\hline & 92 & $5.9 \%$ & $4.5 \%$ & 105 & $7.0 \%$ & $11.0 \%$ \\
& 163 & $43.1 \%$ & $32.8 \%$ & 174 & $10.2 \%$ & $13.8 \%$ \\
Total & 534 & $66.18 \%$ & $61.99 \%$ & & & \\
\hline
\end{tabular}

Fuente: Ibidem.

Nota: El cuadro inferior indica el número de bienes posicionado en cada cuadrante, así como sus respectivas cuotas de mercados.

exportadora de los bienes que han experimentado una mejora en los registros de vU al tiempo que han perdido cuota de mercado.

\section{NOTA FINAL}

A modo de resumen, desde el punto de vista de la calidad de la inserción comercial de la IBE, se aprecian tendencias muy diversas, dependiendo de los indicadores utilizados y de la segmentación de la muestra. Téngase en cuenta, en este sentido que la información estadística utilizada hace referencia a todas las empresas, cualquiera que sea su tamaño, su perfil productivo y tecnológico y el grado de internacionalización de su proceso productivo.

Con todas las cautelas derivadas de la diversidad existente en el seno de la industria, sugiere la existencia de cierta inercia estructural que se traduce, sobre 
todo, en una pérdida de competitividad relativa y un posicionamiento en segmentos de mercado relativamente vulnerables, desde el punto de vista del nivel tecnológico y la escala de gama de nuestras exportaciones. Parece claro que la IBE de la economía espańola se encuentra en una posición relativamente subordina$\mathrm{da}$, mayoritariamente instalada en espacios competitivos de media-baja tecnología y gama. Ello explicaría la intensa presión sobre los precios y el consiguiente ajuste operado en los años de crisis económica.

Los desafíos que enfrentan las empresas y, en términos más generales, la IBE son el producto de la crisis económica, de un conjunto de factores endógenos a la firma y de una problemática estructural de largo recorrido. Esa complejidad en el diagnóstico exige asimismo sofisticación en las políticas aplicadas, más allá de las que persiguen ajustes salariales y de plantilla. Éstos, aun cuando permitan un aumento (mecánico, estadístico) de la productividad del trabajo y ayuden a sanear los beneficios, presentan la restricción (entre otros límites) de actuar sobre los costes y los precios, espacio en el que la competencia es cada vez más intensa.

Grática 14. Valores unitarios y cuotas exportadoras en la Industria de Bienes de Equipo de bajo contenido tecnológico. (Variación entre 2004/2007 y 2008/2009)

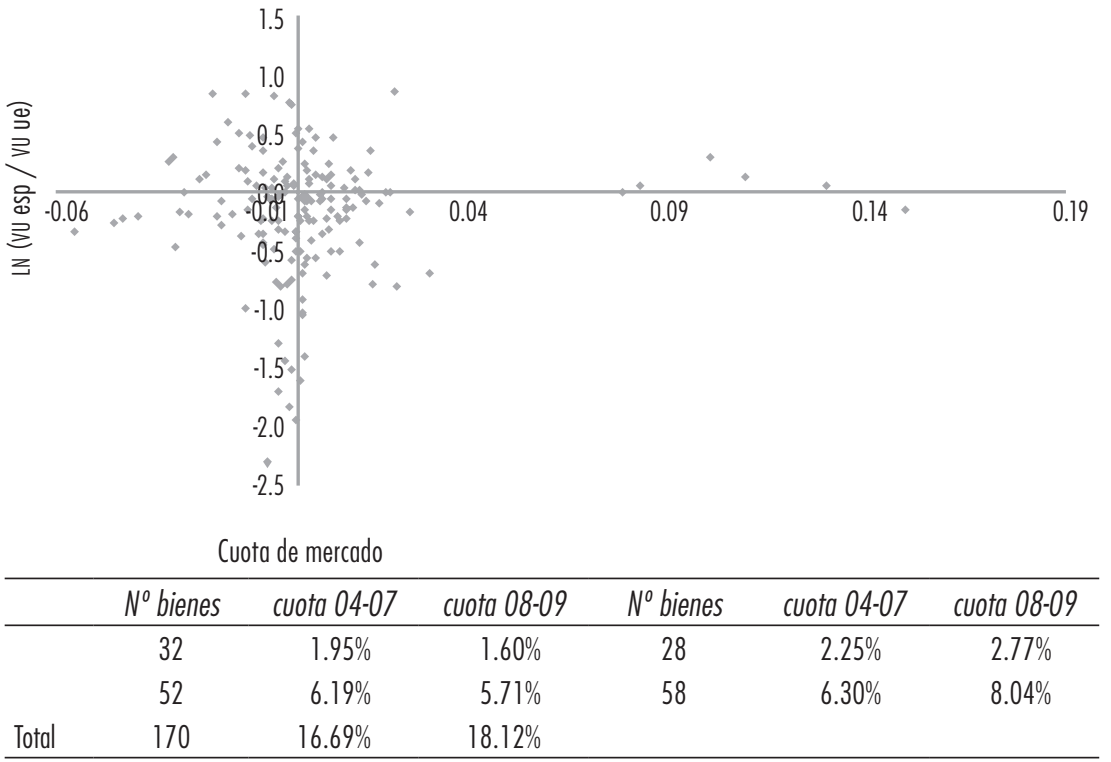

Fuente: Ibidem.

Nota: El cuadro inferior indica el número de bienes posicionado en cada cuadrante, así como sus respectivas cuotas de mercados. 
Fernando Luengo

Lo cierto es que los valores de los indicadores estructurales presentados sugieren la existencia de un potencial de mejora para alcanzar los estándares comunitarios; mejora cuya materialización depende, entre otros factores, de la capacidad inversora y del diseño de un nuevo modelo productivo. Se impone, pues, una intervención estratégica, coherente con el papel vertebrador del sector de medios de producción, su dimensión tecnológica, sus conexiones con la demanda de bienes finales e intermedios, su proyección comercial y su articulación con el capital foráneo.

\section{BIBLIOGRAFÍA}

Aiginger, Karl, "Europe's Position in Quality Competition", European Commission, Enterprise Directorate General, Enterprise DG Working Paper, septiembre 2000 (consultado el 20 de abril de 2010), disponible en <http:// www.pedz.uni-mannheim.de/daten/edz-h/gdb/00/wp_quality.pdf>.

Aiginger, Karl y Stephen Davis, "Industrial Specialisation and Geographic Concentration: Two Sides of the Same Coin? Not for the European Union", Journal of Applied Economics, Vol. vII, num. 2, noviembre 2004.

Beeson, Michael y Iyanatil Islam, "Neoliberalism and East Asia: Resisting the Washington Consensus", Journal of Development Studies, num. 41, 2004.

Commission of the European Communities, "European Competitiveness Report", Commission Staff Working Paper, 2009.

Cooper, Cooler,"Technology, Manufactured Exports and Competitiveness, Global Forum on Industry", Perspectives for 2000 and Beyond, Background Paper, unido, 26 de septiembre 1995.

Davis, Stephen,"Industrial Specialisation and Geographic Concentration: Two Sides of the Same Coin? Not for the European Union", Journal of Applied Economics, Vol. vII, 2004.

Directorate General for Enterprise and Industrie, "Eu Industrial Structure 2009, Performance and Competitiveness", European Commission, 2009.

Economic Commission for Europe, "Technological Activity in the ece Region During the 1990s", Economic Survey of Europe, United Nations, num. 1, 2004.

Fontagné, Lionel et al, "European Industry's Place in the International Division of Labour: Situation and Prospects", CEPII, 2004.

, "Les produits de haute technologie: une source d'asymétrie en Europe", La lettre du CEPII, num. 179, 1999. 
Gambarotto, Francesca y Stefano Solari, "European Capitalisms and Variety of Core-periphery Patterns", 5th International Conference Development in Economic Theory and Policy,10-11 de julio de 2008.

Gaulier, Guillaume y Soledad Zignago, "A World Database of International Trade at the Product-level", CEPII, Working Paper, julio de 2008.

Grossman, Gene M. y Estefan Rossi-Hansberg, "The Rise of Offshoring: It's not Wine for Cloth Anymore", Mimeo, julio de 2006.

Lall, Sanjoy, "Linking, FDI and Technology Development for Capacity Building and Strategic Competitiveness", Transnational Corporations, Vol. 11, num. 3, diciembre de 2002.

Landesmann, Michael, "International Trade and Economic Diversification: Patterns and Policies in the Transition Economies", The Vienna Institute for International Economic Studies (WIIW), Research Reports, num. 350, diciembre, 2009.

Landesmann, Michael. y Julia Wörz, "CEECs Competitiveness in the Global Context", The Vienna Institute for Comparative Economic Studies Research Reports, num. 327, mayo de 2006.

Lapavitsas, Costas, Kaltenbrunner, Aannima et al, "Eurozone Crisis: Beggar Thyself and ty Neyghbour", Research on Money and Finance, RMF Occasional Report, marzo de 2010.

Mulder, Nanno, Rodrigo Paillacar et al, "Market Positioning of Varieties in World Trade: Is Latin America Losig out on Asia?", CEPII, Document de Travail, num. 9, abril de 2009.

Szalavetz, Andrea, "Peripheral Participants in Global Production Networks. Changing Dynamics in the Transformation from Industrial and Intellectual Capitalism", Institute for World Economics. Hungarian Academy of Sciences, Working Papers, num. 142, diciembre de 2003.

United Nations Industrial Development Organization, Structural Changes in Industry and the Global Economy. Breaking in and Moving up: New Industrial Challenges for the Bottom Billion and the Middle-income Countries, Viena, Eds., Anonymous, 2009. 
